\title{
Knowledge-based Consistency Index for Fuzzy Pairwise Comparison Matrices
}

\author{
Sylvain Kubler, William Derigent, Alexandre Voisin, Jérémy Robert, Yves Le Traon
}

\begin{abstract}
Fuzzy AHP is today one of the most used Multiple Criteria Decision-Making (MCDM) techniques. The main argument to introduce fuzzy set theory within AHP lies in its ability to handle uncertainty and vagueness arising from decision makers (when performing pairwise comparisons between a set of criteria/alternatives). As humans usually reason with granular information rather than precise one, such pairwise comparisons may contain some degree of inconsistency that needs to be properly tackled to guarantee the relevance of the result/ranking. Over the last decades, several consistency indexes designed for fuzzy pairwise comparison matrices (FPCMs) were proposed, as will be discussed in this article. However, for some decision theory specialists, it appears that most of these indexes fail to be properly "axiomatically" founded, thus leading to misleading results. To overcome this, a new index, referred to as KCI (Knowledge-based Consistency Index) is introduced in this paper, and later compared with an existing index that is axiomatically well founded. The comparison results show that (i) both indexes perform similarly from a consistency measurement perspective, but (ii) KCI contributes to significantly reduce the computation time, which can save expert's time in some MCDM problems.
\end{abstract}

Index Terms-Analytic hierarchy process (AHP); Fuzzy logic; Multiple criteria decision-making; Consistency; Decision analysis

\section{INTRODUCTION}

$\mathbf{M}$ CDM (Multiple criteria decision-making) methods are frequently used to solve real world problems with multiple, conflicting, and incommensurate criteria and/or objectives. Hwang and Yoon [1] have classified MCDM methods into two categories: multi-attribute decision-making (MADM) and multi-objective decision-making (MODM). MADM techniques, unlike MODM, heavily involve human participation and judgments. Research on human judgments shows that the human brain is able to consider only a limited amount of information at any one time [2], thus making it unreliable to take decisions when facing complex problems. The Analytic Hierarchy Process (AHP), initially introduced by Saaty [3], is by now one of the most widely applied MADM techniques, whose main strength lies in its impartial and logical grading system, as well as its flexibility to be combined with other techniques such as Linear Programming, Genetic Algorithms, Fuzzy Logic, Balanced ScoreCard, etc. [4], [5].

S. Kubler, J. Robert and Y. Le Traon are with the Interdisciplinary Centre for Security, Reliability \& Trust, 4 rue Alphonse Weicker L-2721 Luxembourg (e-mail: firstname.lastname@uni.lu)

A. Voisin and W. Derigent are with the Research Center for Automatic Control of Nancy, Lorraine University, BP 70239, F-54506, Vandœuvre, France (e-mail: firstname.lastname@univ-lorraine.fr)

Copyright (c) 2012 IEEE. Personal use of this material is permitted. However, permission to use this material for any other purposes must be obtained from the IEEE by sending a request to pubs-permissions@ieee.org."
As a practical popular methodology for dealing with uncertainty, the Fuzzy Logic combined with AHP, more commonly known as Fuzzy AHP (FAHP), has found huge applications in recent years [5]. Different from classical set theory, fuzzy set theory permits the gradual assessment of the membership of elements in relation to a set [6]. van Laarhoven and Pedrycz [7] introduced, in 1983, the first FAHP method making use of triangular fuzzy numbers to model uncertainty in comparative judgments. Many other methods were introduced in the years that followed, as reviewed in our recent state-of-the-art survey of FAHP applications [5]. The main argument to introduce fuzzy set within AHP lies in its ability to handle uncertainty and vagueness arising from human judgments. In AHP, such judgments take place when decision makers carry out pairwise comparisons between a set of items (criteria or alternatives). More specifically, decision makers have to specify "how many more times item $i$ is preferred to item $j$ ". As human beings usually reason with granular information rather than precise one, such pairwise comparisons may contain some degree of inconsistency [8]. To overcome this problem, Saaty has introduced a consistency index that enables to quantify the inconsistency of a single pairwise comparison matrix. Nowadays, consistency analyses and indexes can be decomposed into two categories: "intra" and "inter" expert consistency [9]. While the first category focuses on a single decision maker/matrix [10], [11], the second category focuses on inconsistency analyses resulting from a group of decision makers [12], [9]. The focus of our research work is on the first category.

Back to 1985, Buckley [13] proposed a consistency index designed for fuzzy pairwise comparison matrices (FPCMs), which is a straight fuzzification of the Saaty's consistency index. In the years that followed, several-like indexes were introduced, amongst others: (i) the feasible region consistency [14] as well as the discrete region [15]; (ii) the fuzzy preference-programming consistency [16]; (iii) the centric consistency [17]; (iv) the additive consistency [18], [19]; or still (iv) the geometric consistency whose first index was proposed by [20], further adapted in [21], [22]. Despite the advantages and disadvantages of all these indexes, most of the criticisms were raised on their failure to be properly "axiomatically" founded (i.e., based on a well-defined mathematical structure), which may lead to misleading solutions and rankings [23], [24]. For some decision theory specialists, including Saaty [25], it is not even clear that fuzzy sets have ever led to new MCDM methods. Dubois [23] argues that, in many cases, fuzzy sets have just been added to existing techniques (FAHP, fuzzy extensions of ELECTRE, fuzzy weighted averages, etc.) 
with no clear benefits. Dubois argues that fuzzy sets in AHP must be considered, first and foremost, at the "axiomatic" level and not only at the technical computing one. More specifically, the major issue lies in the difficulty to successfully satisfy the transitivity and reciprocal axioms [26], [27], [28], as will be discussed in greater detail in section II. To overcome this, Ohnishi et al. [29] proposed a consistency index - referred to as Ohnishi's index in the rest of this paper - that is axiomatically well founded for FPCMs. In an analogous manner, a new index referred to as "Knowledge-based Consistency Index" (KCI) is proposed and formalized in section III, which is then compared with Ohnishi's index in section IV; the conclusion follows.

The experiment results show that the proposed KCI is, from a consistency measurement perspective, as effective as Ohnishi's index, nonetheless, KCI contributes to significantly reduce the computation time that is an important outcome because it directly impacts on the decision maker's work/activities. Indeed, in traditional MCDM applications, several FPCMs must be carried out by the decision maker (depending on the criteria hierarchy structure), and the time to solve the whole problem (i.e., for obtaining the final alternative ranking) might increase exponentially, as will be presented in the experiment section.

\section{FUZZY AHP: PRINCIPLES, LIMITATIONS \& INDEXES}

We believe that a recap of the main (F)AHP principles must first be given in section II-A, before presenting in section II-B the Ohnishi's index.

\section{A. Fuzzy extension of AHP}

AHP method consists of several stages [4], from the structuration of the problem in a hierarchal structure (composed of the goal, criteria, alternatives), to the pairwise comparisons of these items, whose ideal situation is the following:

1) the pairwise relative preference of $n$ items (alternatives or criteria) is modelled by a $n \times n$ consistent preference matrix $A$, where each coefficient $a_{i j}$ is supposed to reflect how many more times item $i$ is preferred to item $j$;

2) a consistent preference matrix is one that fulfils the reciprocal axiom $\left(a_{j i}=\frac{1}{a_{i j}} \forall i, j\right)$ as well as the transitivity axiom $\left(a_{i j}=a_{i k} \times a_{k j} \forall i, j, k, i \neq k\right)$;

3 ) then, its largest eigenvalue is equal to $n$ and there exists a corresponding eigenvector $\vec{w}=\left(w_{1}, w_{2}, \ldots, w_{n}\right)$ with $\forall i, j, a_{i j}=\frac{w_{i}}{w_{j}}$, yielding relative importance weights.

Even if widely used, AHP has been criticised by some scholars as being ill-founded at the measurement level and, as quoted by Dubois [23]: "Asking for precise values $a_{i j}$ is debatable, because these coefficients are arguably imprecisely known by experts". Several approaches have thus considered fuzzyvalued pairwise comparison data, which consists in extending the computation scheme of Saaty with fuzzy intervals [5]. A fuzzy pairwise comparison number, denoted by $\tilde{a}_{i j}$ in (1), is supposed to reflect the expert preference (item $i$ over $j$ ) with a certain level of imprecision. However, this task turned out to be difficult for several reasons [23]: (i) replacing a consistent preference matrix by a fuzzy-valued preference matrix loses the properties of the former; (ii) fuzzy eigenvalues or vectors of fuzzy-valued matrices are hard to define in a rigorous way; (iii) considering interval-matrix defined from $\alpha$-cuts intervals, denoted by $\tilde{a}_{i j}^{\alpha}=\left[\underline{a}_{i j} ; \bar{a}_{i j}\right]$, leads to other issues (e.g., the boundary matrices are not reciprocal anymore); and so forth.

$$
\tilde{A}=\left[\tilde{a}_{i j}\right]=\begin{gathered}
1 \\
1 \\
2 \\
\vdots \\
n
\end{gathered}\left[\begin{array}{cccc}
\tilde{a}_{11} & \tilde{a}_{12} & \ldots & \tilde{a}_{1 n} \\
\tilde{a}_{21} & \tilde{a}_{22} & \ldots & \tilde{a}_{2 n} \\
\vdots & \vdots & \vdots & \vdots \\
\tilde{a}_{n 1} & \tilde{a}_{n 2} & \ldots & \tilde{a}_{n n}
\end{array}\right]
$$

Instead of viewing fuzzy interval preference matrices as fuzzy substitutes to precise ones, one may on the contrary acknowledge fuzzy pairwise preference data as imprecise knowledge about regular preference information. The fuzzy interval preference matrix is then seen as constraining an ill-known precise consistent comparison matrix. Inconsistencies in comparison data are thus explicitly explained by the imprecise nature of human-originated information. Such a constraint-based view has been aptly explained by Ohnishi et al. [29] who, at the same time, introduced an original approach that is axiomatically well founded to handle inconsistency in FPCMs (i.e. addressing all the abovementioned difficulties). This "constraint-based approach" is presented in more detail in the next section.

\section{B. Constraint-based approach (Ohnishi's index)}

The basic idea behind Ohnishi's approach lies in the fact that the index has to measure the degree to which an AHP-consistent crisp matrix $R$ can fulfill the fuzzy constraints expressed in the FPCM. More concretely, if $\vec{w}=$ $\left(w_{1}, w_{2}, \ldots, w_{n}\right)$ is the weights' vector derived from $R$, the consistency is formulated as in (2):

$$
\alpha(R)=\min _{i j}\left\{\tilde{a}_{i j}\left(\frac{w_{i}}{w_{j}}\right)\right\}
$$

Such an approach is not a one-stage process and requires an optimization stage to find the optimal weights' vector that maximizes $\alpha(R)$. Ohnishi et al. have formulated this optimization problem as a fuzzy constraint satisfaction problem, as formalized in (3), where $n$ is the total number of items ( $c f$. (1)) and $w_{i}$ the weight of item $i$.

$$
\begin{aligned}
& \operatorname{maximize}\left(\alpha=\min _{i j}\left\{\tilde{a}_{i j}\left(\frac{w_{i}}{w_{j}}\right)\right\}\right), 0 \leq w_{i} \leq 1, \\
& i=\{1, . ., n\}, \quad \sum_{i=1}^{n} w_{i}=1
\end{aligned}
$$

Finally, Ohnishi's index (denoted by $\alpha_{\mathrm{Ohn}}$ ) can be formalized as in (4), which is an axiomatically well founded measure that reflects the closeness/distance between the "initial knowledge" expressed by the decision maker in $\tilde{A}$ and the "consistent knowledge" (i.e., the one satisfying the fundamental axioms, as it has been discussed in the previous section). It is important to note that such an index does not correspond, theoretically speaking, to the well known Saaty's consistency index. Nevertheless, it can be considered 
as a natural substitute for evaluating, and most importantly measuring the consistency (or inconsistency) of any FPCM.

$$
\alpha_{\mathrm{Ohn}} \equiv \max _{w_{1}, . ., w_{n}}\left[\min _{i j}\left\{\tilde{a}_{i j}\left(\frac{w_{i}}{w_{j}}\right)\right\}\right]
$$

\section{KNOWLEDGE-BASED CONSISTENCY INDEX}

The formalisation of the problem along with the proposed index $(\mathrm{KCI})$ is presented in section III-A, while the mathematical proof underlying KCI is detailed in section III-B.

\section{A. KCI Formalisation}

In the following, the term "knowledge" about a variable $x$ is used to qualify the set of all possible values that $x$ can take from the expert point of view, each value being associated with a degree of preference. By definition, fuzzy sets are perfectly suited to represent such a knowledge about variables. When considering a FPCM, two different types of knowledge are expressed by the decision maker, namely:

- the direct knowledge related to the comparison of item $i$ over $j$, expressed by $\tilde{a}_{i j}$;

- the indirect knowledge resulting from the transitivity axiom, expressed for each FPCM's item by the fuzzy number(s) $\tilde{a}_{i k} \otimes \tilde{a}_{k j}$.

As Dubois [23] suggests, the crisp transitivity axiom defined as $a_{i j}=a_{i k} \times a_{k j}$ for crisp numbers, does not hold when considering human knowledge since it is granular rather than crisp. A strict equality $(=)$ between fuzzy sets means that all values of $\tilde{a}_{i j}$ would belong to $\tilde{a}_{i k} \otimes \tilde{a}_{k j}$. Obviously, Saaty did not work with sets but with crisp numbers, meaning that the transitivity axiom must not be applied directly, and the above equality rule is too strong and extremely difficult to obtain in practice. Instead of checking for equality between sets, a weaker condition is to verify that pieces of "knowledge" are consistent, meaning that they must have a common element: $\tilde{a}_{i j} \cap\left(\tilde{a}_{i k} \otimes \tilde{a}_{k j}\right)$. When deriving this formulation to all "indirect" items of a FPCM, it leads to:

$$
\left(\cap\left(\tilde{a}_{i k} \otimes \tilde{a}_{k j}\right) \cap \tilde{a}_{i j}\right) \neq \varnothing
$$

Although this condition allows for the verification of compatibility between the direct and indirect knowledge expressed by the DM (i.e., $\tilde{a}_{i j}$ ), it does not provide indicators about the degree of consistency of $\tilde{A}$. Indeed, this condition only returns a binary result, i.e. whether $\tilde{A}$ is or not consistent. Given this observation, we present a new consistency index called Knowledge-based Consistency Index, denoted by $\alpha_{\mathrm{KCI}}$, which is derived from Eq. (5) in the sense that it is based on the inclusion operator rather than the equal or non-disjunction operators. It can be expressed in two different ways:

$$
\begin{gathered}
\cap\left(\tilde{a}_{i k} \otimes \tilde{a}_{k j}\right) \supseteq \tilde{a}_{i j} \\
\forall i, j \in \mathcal{N} \mid i<j \\
\cap\left(\tilde{a}_{i k} \otimes \tilde{a}_{k j}\right) \subseteq \tilde{a}_{i j} \\
\forall i, j \in \mathcal{N} \mid i<j
\end{gathered}
$$

The second formulation is preferred as the multiplication of two fuzzy numbers, i.e. $\left(\tilde{a}_{i k} \otimes \tilde{a}_{k j}\right)$, necessarily introduces an extension of the uncertainty that does not necessary meet the
DM's wish (i.e. $\tilde{a}_{i j}$ ). Then, to quantify the level of consistency of $\tilde{A}$, the inclusion operator $\tilde{\supseteq}$ is defined as in (7).

$$
\begin{aligned}
\tilde{\supseteq}: \tilde{\Re} & \times \tilde{\Re} \longrightarrow[0,1] \\
\tilde{a} \tilde{\supseteq} \tilde{b} & \longrightarrow \sup _{x \in \Re}\left(\min \left(\mu_{\tilde{a}}(x), \mu_{\tilde{b}}(x)\right)\right)
\end{aligned}
$$

Finally, $\alpha_{\mathrm{KCI}}$ can be expressed as the minimum degree to which the DM's knowledge capitalized via the entire FPCM can be satisfied, referring to what extent the transitivity axiom is satisfied ( $c f$. Eq. (6)). All this can be formalized as follows:

$$
\alpha_{\mathrm{KCI}}=\min _{i, j \in \mathcal{N} \mid i<j}\left(\left(\bigcap_{k \in \mathcal{N}-\{i, j\}}\left(\tilde{a}_{i k} \otimes \tilde{a}_{k j}\right)\right) \tilde{\supseteq} \tilde{a}_{i j}\right)
$$

If $0<\alpha_{\mathrm{KCI}}<1$, then $\tilde{A}$ is 'partly' consistent to the level $\alpha$ - although a perfectly crisp consistent matrix in the sense of Saaty can be derived from $\tilde{A}$ - since a compatibility exists between the knowledge expressed by the DM and the transitivity axiom for the $\frac{n(n-1)}{2}$ pairwise comparisons, no matter how small the compatibility is. When $\alpha_{\mathrm{KCI}}=1$, the matrix is considered as 'perfectly' consistent, meaning that direct knowledge is completely included in indirect knowledge. However, if $\alpha_{\mathrm{KCI}}=0, \tilde{A}$ can be reported as 'fully' inconsistent because, for one of the $\frac{n(n-1)}{2}$ pairwise comparisons, the DM knowledge is not compatible/included with the knowledge resulting from the transitivity axiom operation.

Deriving a crisp consistent matrix from a fuzzy one is often a subject of debate because no explicit mathematical demonstration is provided to express the link between fuzzy and crisp consistency indices. To avoid this debate, the following section demonstrates, based on mathematical proof, the relation between $\alpha_{\mathrm{KCI}}$ and the consistency of a crisp matrix.

\section{B. Proof of relation between $\alpha_{K C I}$ and crisp matrix consistency}

Let $\tilde{A}$ be a fuzzy triangular matrix, and $A^{\alpha}=\left[\tilde{a}_{i j}^{\alpha}\right]$ the $\alpha$-cut matrix of $\tilde{A}$ referred to as alpha-matrix, i.e. a matrix composed of the $\alpha$-cuts of the each item of $\tilde{A}$.

Definition 1 (alpha-matrix consistency). $A^{\alpha}$ is consistent if and only if (9) is satisfied. All $w_{i}$ respecting this inequation is called the "feasible region" [14].

$$
\exists w_{i}, w_{j} \in \mathbb{R}^{+}, \underline{a}_{i j}^{\alpha} \leq \frac{w_{i}}{w_{j}} \leq \bar{a}_{i j}^{\alpha}, \forall \tilde{a}_{i, j}
$$

In fact, this definition of consistency for interval matrices is similar to the one introduced in [30], as recalled in Theorem 1. Indeed, this definition means that a vector $\vec{w}=\left(w_{1}, w_{2}, \ldots, w_{n}\right)$ can be derived from $A^{\alpha}$, which can be seen as an eigenvector of a perfectly-consistent crisp matrix whose values $a_{i j}$ are always between $\underline{a}_{i j}^{\alpha}$ and $\bar{a}_{i j}^{\alpha}$ :

Theorem 1 (alpha-matrix consistency check). $A^{\alpha}$ is a consistent matrix if, and only if:

$$
\max _{k}\left(\underline{a}_{i j}^{\alpha} ; \underline{a}_{i k}^{\alpha} \times \underline{a}_{k j}^{\alpha}\right) \leq \min _{k}\left(\bar{a}_{i j}^{\alpha} ; \bar{a}_{i k}^{\alpha} \times \bar{a}_{k j}^{\alpha}\right), \forall i, j, k
$$


Proof. Following Definition 1, if $A^{\alpha}$ is a consistent matrix then the feasible region is not empty, which means that there is no contradiction among the following inequality constraints:

$$
\begin{array}{r}
\underline{a}_{i k}^{\alpha} \leq w_{i} / w_{k} \leq \bar{a}_{i k}^{\alpha}, i, k=1, \ldots, n \\
\underline{a}_{k j}^{\alpha} \leq w_{k} / w_{j} \leq \bar{a}_{k j}^{\alpha}, k, j=1, \ldots, n \\
\underline{a}_{i j}^{\alpha} \leq w_{i} / w_{j} \leq \bar{a}_{i j}^{\alpha}, i, j=1, \ldots, n
\end{array}
$$

Multiplying (11) by (12) leads to the implicit inequality:

$$
\underline{a}_{i k}^{\alpha} \times \underline{a}_{k j}^{\alpha} \leq w_{i} / w_{j} \leq \bar{a}_{i k}^{\alpha} \times \bar{a}_{k j}^{\alpha}, i, j, k=1, \ldots, n
$$

(13) and (14) imply the following inequality :

$$
\max \left(\underline{a}_{i j}^{\alpha} ; \underline{a}_{i k}^{\alpha} \times \underline{a}_{k j}^{\alpha}\right) \leq w_{i} / w_{j} \leq \min \left(\bar{a}_{i j}^{\alpha} ; \bar{a}_{i k}^{\alpha} \times \bar{a}_{k j}^{\alpha}\right)
$$

Since (15) holds for any $k=\{1, \ldots, n\}$, it follows that $\max _{k}\left(\underline{a}_{i j}^{\alpha} ; \underline{a}_{i k}^{\alpha} \times \underline{a}_{k j}^{\alpha}\right) \leq \min _{k}\left(\bar{a}_{i j}^{\alpha} ; \bar{a}_{i k}^{\alpha} \times \bar{a}_{k j}^{\alpha}\right)$ holds for all $i, j, k=1, \ldots, n$.

Theorem 2 (relation between knowledge and alpha-matrix consistency). If $\tilde{A}$ is a knowledge-based consistent matrix to the $\alpha$-level (measured by $\alpha_{K C I}$ in this paper), $A^{\alpha}$ is a consistent matrix.

Proof. As previously detailed in (5) and 6, $\tilde{A}$ is a knowledgebased consistent FPCM matrix if, and only if, the following equation holds for all its items $\tilde{a}_{i j}$ :

$$
\tilde{c}_{i j}=\left(\tilde{a}_{i j} \cap\left(\tilde{a}_{i k} \otimes \tilde{a}_{k j}\right)\right) \neq \oslash, \forall \underset{i<k<j}{i, j, k}=\{1, \ldots, n\}
$$

Applying alpha-cuts on (16) would result in the following expressions:

$$
\begin{array}{r}
\tilde{c}_{i j}=\tilde{a}_{i j} \cap\left(\tilde{a}_{i k} \otimes \tilde{a}_{k j}\right) \\
\Rightarrow c_{i j}^{\alpha}=a_{i j}^{\alpha} \cap\left(a_{i k}^{\alpha} \times a_{k j}^{\alpha}\right) \\
\Rightarrow\left[\underline{c}_{i j}^{\alpha} ; \bar{c}_{i j}^{\alpha}\right]=\left[\underline{a}_{i j}^{\alpha} ; \bar{a}_{i j}^{\alpha}\right] \cap\left(\left[\underline{a}_{i k}^{\alpha} ; \bar{a}_{i k}^{\alpha}\right] \times\left[{ }^{\alpha} \underline{a}_{k j}^{\alpha} ;{ }^{\alpha} \bar{a}_{k j}^{\alpha}\right]\right) \\
\Rightarrow\left[\underline{c}_{i j}^{\alpha} ; \bar{c}_{i j}^{\alpha}\right]=\left[\underline{a}_{i j}^{\alpha} ; \bar{a}_{i j}^{\alpha}\right] \cap\left[\underline{a}_{i k}^{\alpha} \times \underline{a}_{k j}^{\alpha} ; \bar{a}_{i k}^{\alpha} \times \bar{a}_{k j}^{\alpha}\right] \\
\Rightarrow\left[\underline{c}_{i j}^{\alpha} ; \bar{c}_{i j}^{\alpha}\right]=\left[\max \left(\underline{a}_{i j}^{\alpha} ; \underline{a}_{i k}^{\alpha} \times \underline{a}_{k j}^{\alpha}\right) ; \min \left(\bar{a}_{i j}^{\alpha} ; \bar{a}_{i k}^{\alpha} \times \bar{a}_{k j}^{\alpha}\right)\right]
\end{array}
$$

where the last equation is equivalent to the alpha-matrix consistency check theorem (i.e., Theorem 1). This means that when, for a fuzzy matrix $\tilde{A}$, knowledge-based consistency at level $\alpha$ is reached, the alpha-matrix $A^{\alpha}$ is also consistent. Given this, it is always possible to derive a consistent crisp matrix from a FPCM matrix with $\alpha_{\mathrm{KCI}}>0$ (if $\alpha_{\mathrm{KCI}}=0$, it is simply not possible to derive a crisp matrix satisfying the transitivity axiom).

As a conclusion, our index allows for detecting an inconsistency (similarly to the C.I. proposed by Saaty), but also for checking and quantifying the "knowledge-based consistency" by evaluating to what extent the DM's knowledge is satisfied.

\section{KCI IMPLEMENTATION \& EVALUATION}

In order to make the understanding of the proposed consistency index $\left(\alpha_{\mathrm{KCI}}\right)$ easier, section IV-A details in a "graphical" manner the different computation stages, while section IV-B focuses on the comparison study: $\alpha_{\mathrm{KCI}} v s$. $\alpha_{\mathrm{Ohn}}$.

\section{A. Use case definition \& KCI implementation}

To illustrate the $\alpha_{\mathrm{KCI}}$ computing stages, let us consider a triangular FPCM from the literature, namely from [31] in which an expert has carried out pairwise comparisons between four emergency response capacities, which are respectively denoted by $\mathrm{C} 1, \mathrm{C} 2, \mathrm{C} 3$ and $\mathrm{C} 4$ in $\tilde{A}$ in Eq. (22).

$$
\begin{aligned}
& \begin{array}{lllll}
\mathrm{C} 1 & \mathrm{C} 2 & \mathrm{C} 3 & \mathrm{C} 4
\end{array} \\
& \tilde{A}=\begin{array}{c}
\mathrm{C} 1 \\
\mathrm{C} 3 \\
\mathrm{C} 4
\end{array}\left[\begin{array}{cccc}
{[1,1,1]} & {\left[\frac{3}{2}, 2, \frac{5}{2}\right]} & {\left[\frac{2}{3}, 1,2\right]} & {\left[1, \frac{3}{2}, 2\right]} \\
\tilde{a}_{21}^{-1} & {[1,1,1]} & {\left[\frac{2}{3}, 1,2\right]} & {\left[\frac{1}{2}, \frac{2}{3}, 1\right]} \\
\tilde{a}_{31}^{-1} & \tilde{a}_{32}^{-1} & {[1,1,1]} & {\left[\frac{1}{2}, \frac{2}{3}, 1\right]} \\
\tilde{a}_{41}^{-1} & \tilde{a}_{42}^{-1} & \tilde{a}_{43}^{-1} & {[1,1,1]}
\end{array}\right]
\end{aligned}
$$

$\tilde{A}$ is graphically displayed in Fig. 1, where the blue/solid membership functions correspond to the $\tilde{a}_{i j}$ items, and the $\mathrm{red} /$ dashed ones to the membership functions resulting from the transitivity axiom operation $\left(\tilde{a}_{i j} \otimes \tilde{a}_{j k}\right)$. Note that more than one fuzzy number result from this operation since $\operatorname{dim}(\tilde{\mathcal{A}})>3$. Considering $\tilde{a}_{12}$ and $\alpha=0$, the following result is obtained when applying Theorem 1 (i.e., (10)):

$$
\begin{aligned}
& \max \left(\underline{a}_{12}^{\alpha} ;\left(\begin{array}{c}
\underline{a}_{13}^{\alpha} \times \underline{a}_{32}^{\alpha} \\
\underline{a}_{14}^{\alpha} \times \underline{a}_{42}^{\alpha}
\end{array}\right)\right) \leq \min \left(\bar{a}_{12}^{\alpha} ;\left(\begin{array}{c}
\bar{a}_{13}^{\alpha} \times \bar{a}_{32}^{\alpha} \\
\bar{a}_{14}^{\alpha} \times \bar{a}_{4}^{\alpha} 2
\end{array}\right)\right) \\
& \max \left(\frac{3}{2} ;\left(\begin{array}{c}
2 / 3 \times 1 / 2 \\
1 \times 1
\end{array}\right)\right) \leq \min \left(\frac{5}{2} ;\left(\begin{array}{c}
2 \times 3 / 2 \\
2 \times 2
\end{array}\right)\right) \\
& \frac{3}{2} \leq \frac{5}{2}
\end{aligned}
$$

The in-equation is satisfied, meaning that $c_{12}^{0}$ is consistent; more specifically, $\frac{3}{2}$ and $\frac{5}{2}$ respectively correspond to the lower and upper interval values of the "support" of $\tilde{c}_{12}$, which corresponds to the yellow/meshed shape $\mathrm{C}_{1,2}$ in Fig. 1. If we now look at the support interval of all the other yellow/meshed shapes in Fig. 1 (i.e., $\forall i, j$ ), it can be observed that Theorem 1 is satisfied for the whole matrix since all in-equations are satisfied.

Given this conclusion, the KCI score can be computed based on Theorem 2. To this end, all items $\tilde{c}_{i j}$ are first computed, which correspond to the different yellow/meshed shapes that have resulted from both the intersection of $\tilde{a}_{i j}$ and $\tilde{a}_{i k} \otimes \tilde{a}_{k j}$ and its inclusion within $\tilde{a}_{i j}$ (cf. (8)). As a second and final step, $\alpha_{\mathrm{KCI}}$ is computed, which is expressed as the minimal vertex/top value of the different yellow/meshed shapes (a vertex/top value being graphically symbolized with $*$ in Fig. 1). Applying (8) along with the $*$ values, the following $\alpha_{\mathrm{KCI}}$ is obtained:

$$
\begin{aligned}
\alpha_{\mathrm{KCI}} & =\min \left(\sup \left(\tilde{c}_{12}\right), \sup \left(\tilde{c}_{13}\right), \ldots, \sup \left(\tilde{c}_{34}\right)\right) \\
& =\min (0.55,0.47,0.49,0.53,0.88,0.42) \\
& =0.42
\end{aligned}
$$

As a conclusion, $\tilde{A}$ is KCI-consistent of value 0.42 , bearing in mind that: "the higher the $\alpha_{K C I}$ score $(\in[0 ; 1])$, the more axiomatically consistent the initial knowledge entered by the expert, and the more satisfied this expert will be." If $\alpha_{\mathrm{KCI}}>0$, the expert's knowledge is consistent in the sense of Saaty since there exists a crisp consistent pairwise comparison matrix. 


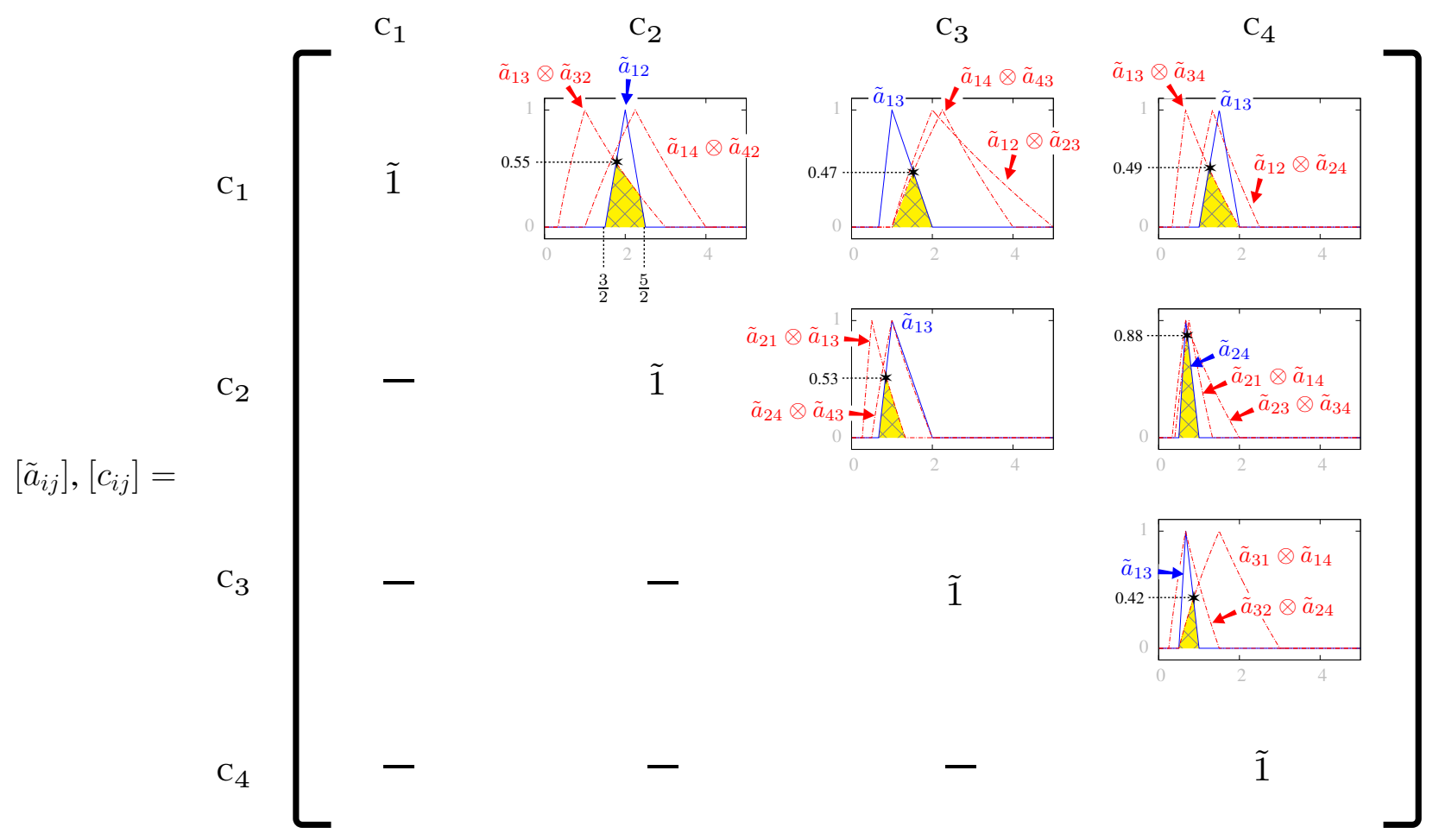

Fig. 1. $4 \times 4$ FPCM (Fuzzy Pairwise Comparison Matrix) specified by an expert - in [31] - for emergency response capacity assessment.

TABLE I

EXPERIMENT DATA \& OUTCOMES: OHNISHI'S INDEX $v s$. KCI

\begin{tabular}{|c|c|c|c|c|}
\hline Size & Set of FPCMs/References selected from the online FAHP testbed ${ }^{1}$ & Score similarity & Consistent FPCMs & Time difference (in $s$ ) \\
\hline $3 \times 3$ & $\begin{array}{l}\text { \{Demirel2012b, Yucenur2011b, Isaai2011a, BuyukozkanCII2007b, Toklu2016, } \\
\text { Sabaghi2016c, Yu2015a, Bereketli2013a, cSen2010b, Duran2008\} }\end{array}$ & $100 \%(\Delta=0.10 \%)$ & $100 \%$ & {$[0.97 ; 7.66]$} \\
\hline $4 \times 4$ & $\begin{array}{l}\text { \{Beskese2015, Lin2010, ChouAiC2013, Ju2012a, Nezarat2015a, Yuksel2010a, } \\
\text { Singh2015, LeeESwA2008, Chan2013a, Lee2009a }\end{array}$ & $100 \%(\Delta=0.01 \%)$ & $50 \%$ & {$[2.44 ; 10.5]$} \\
\hline $5 \times 5$ & $\begin{array}{l}\text { \{Yu2015b, Efe2016, cSen2010a, Chan2008, Yang2010, LeeESA2009, Le2016c, } \\
\text { Kannan2013, Das2015, cSen2010a }\end{array}$ & $100 \%(\Delta=0.05 \%)$ & $30 \%$ & {$[3.65 ; 25.33]$} \\
\hline $7 \times 7$ & $\begin{array}{l}\text { \{Calabrese2013, Prakash2016, Kabak2014, Ren2014, Jakiel2015, Zolfani2012, } \\
\text { Rostamzadeh2011a, Cebeci2009b, Rostamzadeh2011, HosseiniFirouz2015 }\end{array}$ & $100 \%(\Delta=0.09 \%)$ & $20 \%$ & {$[10.14 ; 72.37]$} \\
\hline$\geq 10 \times 10$ & $\begin{array}{l}\text { \{Bozbura2007, Nguyen2014, Ren2014b, Ren2014c, Taha2011a, Akkaya2015, } \\
\text { Govindan2015, Routroy2013\} }\end{array}$ & $100 \%(\Delta=0.10 \%)$ & $20 \%$ & {$[36.68 ; 299.0]$} \\
\hline
\end{tabular}

\section{B. Comparison study: KCI vs. Ohnishi's index}

The two indexes (i.e., $\alpha_{\mathrm{KCI}}$ and $\alpha_{\mathrm{Ohn}}$ ) have been implemented for comparison purposes under the MATLAB environment (version R2014b) and tested on an Intel Pentium Core i7-2677m machine with 1.80 GHz CPU and 4GB memory. Parameters considered for this comparison study are (i) "similarity" of the scores between $\alpha_{\mathrm{KCI}}$ and $\alpha_{\mathrm{Ohn}}$; and (ii) "computation time" required by each index. To this end, several FPCMs from the literature have been selected from the online FAHP testbed ${ }^{2}$ released in our recent state-of theart survey [5]. In an effort to carry out an "heterogeneous" comparison study (i.e., considering FPCMs whose size varies), about ten matrices per size category (from $3 \times 3$ to $\geq 10 \times 10$ ) have been selected, as summarized in TABLE I. First results and findings have been also reported in this table, as it is discussed in the following.

An important outcome regarding the first comparison criterion - i.e., how "similar" the index scores are? - is that

${ }^{2}$ Official testbed URL: http://fahptestbed.sntiotlab.lu both scores are identical in $100 \%$ of the cases (maximum deviation of $0.1 \%$ ). To put it another way, $\alpha_{\mathrm{KCI}}$ is, from a consistency measurement viewpoint, as efficient as $\alpha_{\text {Ohn }}$ index and vice-versa. The proportion of consistent FPCMs amongst the ten matrices considered in each size category is also reported in TABLE I ( $c f .4^{\text {th }}$ column). Results show that $100 \%$ of the $3 \times 3$ matrices are consistent according to the two index definitions (i.e., $\alpha_{\mathrm{Ohn}}=\alpha_{\mathrm{KCI}}>0$ ), while this trend is decreasing along with the increase of the matrix size (only $20 \%$ of $7 \times 7$ and $\geq 10 \times 10$ FPCMs being consistent). This result somehow confirms that the higher the number of criteria to be compared in a pairwise manner, the more difficult it becomes for the human brain. Having said that, it would be interesting to have a more in-depth look at the consistency score obtained for the consistent FPCMs, e.g. regarding the $30 \%$ of $5 \times 5$ consistent FPCMs (i.e., 3 out of the 10 evaluated). Fig. 2(a) provides such an overview, where the min and max consistency scores of those FPCMs are displayed. No specific pattern behaviour can be identified through this histogram, except that the average consistency score is around 0.4 for 


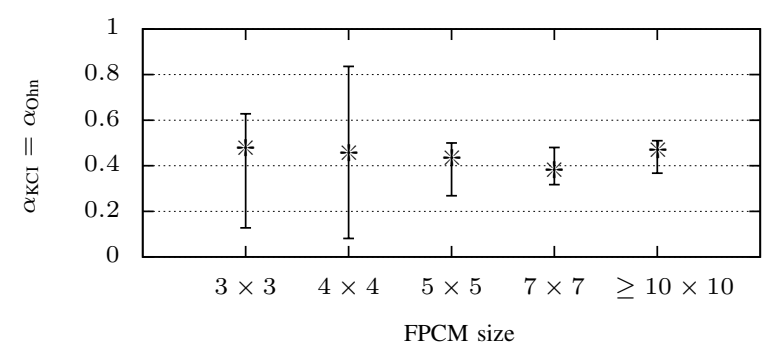

(a) Min-Max consistency scores obtained from both approaches

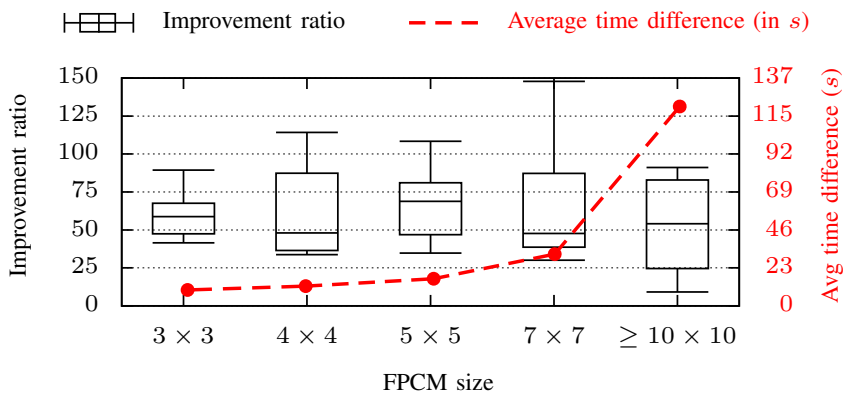

(b) "Computation Time" comparison study

Fig. 2. Comparison study "KCI vs. Ohnishi's index" from an efficiency and time computation standpoint

all matrix sizes (cf. $*$ in Fig. 2(a)) and that the standard deviation of the consistency scores decreases over the increase of the matrix size. Nonetheless, this finding needs to be put into perspective because only a small proportion of consistent matrices in the upper size range were considered, e.g. only two FPCMs out of the ten $7 \times 7$ matrices proved to be consistent (i.e., $\alpha_{\mathrm{Ohn}}=\alpha_{\mathrm{KCI}}>0$ ). In an extended version of this paper, the whole set of FPCMs that is today's available on the online FAHP testbed will be considered (255 matrices in total) so as to confirm or reject these initial findings.

The second aspect of our comparison study is looking at the computation time required by both indexes. Such an analysis is provided in Fig. 2(b), in which two complementary graphs/information are displayed:

- Improvement ratio, $\left[T_{\alpha_{\mathrm{Ohn}}} / T_{\alpha_{\mathrm{KCI}}}\right]$ : the higher the ratio, the more efficient our approach is over Ohnishi;

- Average time difference, avg $\left(\left[T_{\alpha_{\mathrm{Ohn}}}-T_{\alpha_{\mathrm{KCI}}}\right]\right)$ : average of the differences between the computation times required by $\alpha_{\mathrm{Ohn}}$ and $\alpha_{\mathrm{KCI}}$ for the set of FPCMs per category. It means that the higher the average time difference, the more meaningful/significant the improvement ratio is. For example, an improvement ratio of 20 is much less significant if we are dealing with milliseconds (e.g., if $T_{\alpha_{\mathrm{Ohn}}}=1 \mathrm{~ms}$, then $\left.T_{\alpha_{\mathrm{KCI}}}=\frac{1 m s}{20}=0.05 \mathrm{~ms}\right)$ than with minutes (e.g., if $T_{\alpha_{\mathrm{Ohn}}}=5 \mathrm{~min}$, then $T_{\alpha_{\mathrm{KCI}}}=\frac{5 \min }{20}=15 \mathrm{~s}$ ).

Looking first at the improvement ratio boxplots in Fig. 2(b), it is clear that $\alpha_{\mathrm{KCI}}$ always performs faster than $\alpha_{\mathrm{Ohn}}$ since, in $50 \%$ of the cases (values between the $1^{\text {st }}$ and $3^{\text {rd }}$ quartiles) our approach performs $\times 25$ to $\times 80$ faster than Ohnishi's approach, and up to $\times 80$ to $\times 150$ in $25 \%$ of the cases (values/cases between the $3^{\text {rd }}$ quartile and the maximum value). Looking now at the "significance" of these results, i.e. inferring these results with the average time difference (red curve in Fig. 2(b)), it appears that the computation time is impacted when the FPCM size increases. For example, the computation time difference between our approach and Ohnishi's one is $\approx 20 s$ when dealing with $5 \times 5$ FPCMs, lasting up to almost $2 \min$ ( $117 \mathrm{~s}$ to be exact) when dealing with $\geq 10 \times 10$ FPCMs. One of the main reasons behind such an increase of time difference is that, unlike KCI, Ohnishi's index requires an optimization stage (see section II-B) where

\footnotetext{
${ }^{2}$ The reader can found/access the scientific article that is related to each "label" given in TABLE I (e.g., Demirel2012b) via the online FAHP testbed.
}

TABLE II

EXPERIMENT DATA \& OUTCOMES: OHNISHI'S INDEX vs. KCI

\begin{tabular}{|c|c|c|c|c|c|c|}
\hline \multirow[b]{2}{*}{ Reference } & \multicolumn{5}{|c|}{ Number of FPCMs carried out in the study } & \multirow{2}{*}{$\begin{array}{l}\text { Additional } \\
\text { Time }\end{array}$} \\
\hline & $3 \times 3$ & $4 \times 4$ & $5 \times 5$ & $7 \times 7$ & $>10 \times 10$ & \\
\hline$\overline{\text { Efe2016 }}$ & 1 & 16 & 2 & 0 & 0 & $2^{\prime} 00^{\prime \prime}$ \\
\hline LeeESA2009 & 3 & 1 & 38 & 0 & 0 & $8^{\prime} 30^{\prime \prime}$ \\
\hline Hosseini...2015 & 2 & 0 & 0 & 6 & 0 & $2^{\prime} 40^{\prime \prime}$ \\
\hline Ren2014b & 0 & 0 & 0 & 0 & 7 & $13^{\prime} 30^{\prime \prime}$ \\
\hline Akkaya2015 & 0 & 0 & 0 & 10 & 1 & $6^{\prime} 00^{\prime \prime}$ \\
\hline Bozbura2007 & 2 & 0 & 0 & 0 & 7 & $13^{\prime} 40^{\prime \prime}$ \\
\hline$\ldots$ & $\ldots$ & $\ldots$ & $\ldots$ & $\ldots$ & . & .. \\
\hline
\end{tabular}

the solution space becomes progressively more complicated (larger) as the FPCM size increases. This time difference gap between both approaches might become even much higher when considering a wider range of $\geq 10 \times 10 \mathrm{FPCMs}$. Indeed, our study did not consider FPCMs whose size is $>13 \times 13$, while some studies consider bigger FPCMs ${ }^{3}$.

It is also important to point out the fact that, in a traditional FAHP study, more than one single FPCM must be carried out by decision maker(s) depending on how many criteria levels, sub-levels, and alternatives compose the hierarchy. This has, obviously, a non negligible impact on the overall time spent by those decision maker(s) to fulfil the FPCMs-related task. Just to provide a very rough estimate of how time-consuming it could become, we randomly selected (from TABLE I) a subset of references/papers for which we emphasize in TABLE II:

- how many FPCMs have to be carried out by the decision maker with regard to each MCDM problem, and;

- what additional time would be necessary for the decision maker to handle all FPCMs in cases he/she would use Ohnishi's index instead of KCI.

This is a "rough estimate" because we took into account the average time difference (per size category) reported in Fig. 2(b) for computing the estimated additional time; e.g., considering "Akkaya2015", the following estimate has been made: $(10 \times 26 s)+(1 \times 117 s)=377 s \approx 6^{\prime} 00 "$. When considering the MCDM problem as a whole, this brief table shows that it can become quite time consuming to deal with consistency in all FPCMs (e.g., $\approx 13^{\prime} 00$ " for "Ren2014b") which, as stated in the introduction section, can impact on the decision maker's daily activities (i.e., need to wait for several minutes to obtain the final alternative ranking).

\footnotetext{
${ }^{3}$ The reader can refer to our online FAHP testbed to identify such studies.
} 


\section{CONCLUSION}

Fuzzy Logic combined with AHP, also referred to as Fuzzy AHP, has been introduced to handle uncertainty and vagueness arising from human judgments, specifically when decision makers carry out pairwise comparisons between items. Such a combination does not come without theoretical problems, especially regarding how to assess and handle (in)consistency in FPCMs. It is natural for people to want to be consistent, which is often thought of as a prerequisite to clear thinking. Over the last two decades, several consistency indexes were introduced to overcome this challenge. However, despite their advantages and disadvantages, most of the criticisms were raised on their failure to be properly "axiomatically" founded. From the reviewed literature, the consistency index introduced by Ohnishi et al. [29] is, to the best of our knowledge, the most axiomatically well founded index and, as a result, is considered in our study as a reference index.

This paper introduces a new index, referred to as $\mathrm{KCI}$ (Knowledge-based Consistency Index), which is axiomatically well founded and intended to help decision makers to measure how distant their knowledge (i.e., the one specified in a FPCM) is from the consistent knowledge (i.e., the one satisfying the fundamental axioms). KCI does not correspond, theoretically speaking, to Saaty's index but can be seen as a natural substitute for evaluating and measuring the degree of (in)consistency in triangular FPCMs: "the higher the $\mathrm{KCI}$ score $(\in[0 ; 1])$, the more axiomatically consistent the initial knowledge entered by the expert". The proposed KCI is further compared with Ohnishi's index, whose results show that (i) both indexes perform similarly from a consistency measurement perspective, but (ii) KCI contributes to significantly reduce the computation time, which can help experts to save time considering the whole MCDM problem. In future research work, this comparison study will be extended to consider a much wider range of FPCMs so as to confirm these findings, and potentially identify other improvement aspects.

\section{ACKNOWLEDGMENT}

The research leading to this publication is supported by the EU's H2020 Programme for research, technological development and demonstration (grant 688203), as well as the National Research Fund Luxembourg (grant 9095399).

\section{REFERENCES}

[1] C. L. Hwang and K. Yoon, Multiple Attribute Decision-Making Methods and Applications. Springer Verlag, Berlin, Heidelberg, New York, 1981.

[2] L. Simpson, "Do decision makers know what they prefer?: Mavt and electre ii," Journal of the Operational Research Society, vol. 47, no. 7, pp. 919-929, 1996.

[3] T. L. Saaty, The Analytic Hierarchy Process. New York: McGraw-Hill, 1980.

[4] O. Vaidya and S. Kumar, "Analytic hierarchy process: An overview of applications," European Journal of operational research, vol. 169, no. 1, pp. 1-29, 2006.

[5] S. Kubler, J. Robert, W. Derigent, A. Voisin, and Y. Le Traon, "A stateof the-art survey \& testbed of fuzzy ahp (fahp) applications," Expert Systems with Applications, vol. 65, pp. 398-422, 2016.

[6] L. Zadeh, "Fuzzy sets," Information and Control, vol. 8, pp. 338-353, 1965.

[7] P. van Laarhoven and W. Pedrycz, "A fuzzy extension of saaty's priority theory," Fuzzy Sets and Systems, vol. 11, no. 1, pp. 199-227, 1983.
[8] R. Urena, F. Chiclana, and E. Herrera-Viedma, "Consistency based completion approaches of incomplete preference relations in uncertain decision contexts," in IEEE International Conference on Fuzzy Systems (FUZZ-IEEE), 2015, pp. 1-6.

[9] M. Brunelli and M. Fedrizzi, "Boundary properties of the inconsistency of pairwise comparisons in group decisions," European Journal of Operational Research, vol. 240, no. 3, pp. 765-773, 2015.

[10] P. Grošelj and L. Z. Stirn, "Acceptable consistency of aggregated comparison matrices in analytic hierarchy process," European Journal of Operational Research, vol. 223, no. 2, pp. 417-420, 2012.

[11] F. Liu, W.-G. Zhang, and Z.-X. Wang, "A goal programming model for incomplete interval multiplicative preference relations and its application in group decision-making," European Journal of Operational Research, vol. 218, no. 3, pp. 747-754, 2012.

[12] D. J. Weiss and J. Shanteau, "The vice of consensus and the virtue of consistency," Psychological investigations of competent decision making, pp. 226-240, 2004.

[13] J. Buckley, "Fuzzy hierarchical analysis," Fuzzy Sets and Systems, vol. 17, pp. 233-247, 1985.

[14] A. A. Salo, "On fuzzy ratio comparisons in hierarchical decision models," Fuzzy Sets and Systems, vol. 84, no. 1, pp. 21-32, 1996.

[15] H. Zhang, Q. Zheng, T. Liu, and Y. Nan, "A new approach to improve the consistency of linguistic pair-wise comparison matrix and derive interval weight vector," in 2014 IEEE International Conference on Fuzzy Systems (FUZZ-IEEE), 2014, pp. 1190-1196.

[16] Y.-M. Wang and K.-S. Chin, "A linear programming approximation to the eigenvector method in the analytic hierarchy process," Information Sciences, vol. 181, no. 23, pp. 5240-5248, 2011.

[17] E. Bulut, O. Duru, T. Keçeci, and S. Yoshida, "Use of consistency index, expert prioritization and direct numerical inputs for generic fuzzy-ahp modeling: A process model for shipping asset management," Expert Systems with Applications, vol. 39, no. 2, pp. 1911-1923, 2012.

[18] Z.-J. Wang, "Derivation of intuitionistic fuzzy weights based on intuitionistic fuzzy preference relations," Applied Mathematical Modelling, vol. 37, no. 9, pp. 6377-6388, 2013.

[19] J. Chu, X. Liu, and Z. Gong, "Two decision making models based on newly defined additively consistent intuitionistic preference relation," in IEEE International Conference on Fuzzy Systems (FUZZ-IEEE), 2015, pp. 1-8.

[20] G. Crawford and C. Williams, "A note on the analysis of subjective judgment matrices," Journal of mathematical psychology, vol. 29, no. 4, pp. 387-405, 1985.

[21] J. Aguaron and J. M. Moreno-Jiménez, "The geometric consistency index: Approximated thresholds," European Journal of Operational Research, vol. 147, no. 1, pp. 137-145, 2003.

[22] J. Ramík and P. Korviny, "Inconsistency of pair-wise comparison matrix with fuzzy elements based on geometric mean," Fuzzy Sets and Systems, vol. 161, no. 11, pp. 1604-1613, 2010.

[23] D. Dubois, "The role of fuzzy sets in decision sciences: Old techniques and new directions," Fuzzy Sets and Systems, vol. 184, no. 1, pp. 3-28, 2011.

[24] F. Meng and X. Chen, "A new method for triangular fuzzy compare wise judgment matrix process based on consistency analysis," International Journal of Fuzzy Systems, pp. 1-20, 2016.

[25] T. L. Saaty and L. T. Tran, "On the invalidity of fuzzifying numerical judgments in the analytic hierarchy process," Mathematical and Computer Modelling, vol. 46, no. 7-8, pp. 962-975, 2007.

[26] Y.-M. Wang, Y. Luo, and Z. Hua, "On the extent analysis method for fuzzy ahp and its applications," European Journal of Operational Research, vol. 186, no. 2, pp. 735-747, 2008.

[27] Y.-M. Wang and K.-S. Chin, "Fuzzy analytic hierarchy process: A logarithmic fuzzy preference programming methodology," International Journal of Approximate Reasoning, vol. 52, no. 4, pp. 541-553, 2011.

[28] K. Zhü, "Fuzzy analytic hierarchy process: Fallacy of the popular methods," European Journal of Operational Research, vol. 236, no. 1, pp. 209-217, 2014.

[29] S.-I. Ohnishi, D. Dubois, H. Prade, and T. Yamanoi, "A fuzzy constraintbased approach to the analytic hierarchy process," in Uncertainty and intelligent information systems. World Scientific, 2008, pp. 217-228.

[30] Y.-M. Wang, J.-B. Yang, and D.-L. Xu, "Interval weight generation approaches based on consistency test and interval comparison matrices," Applied Mathematics and Computation, vol. 167, no. 1, pp. 252-273, 2005.

[31] Y. Ju, A. Wang, and X. Liu, "Evaluating emergency response capacity by fuzzy ahp and 2-tuple fuzzy linguistic approach," Expert Systems with Applications, vol. 39, no. 8, pp. 6972-6981, 2012. 